\section{REPORTING ETHICAL PROCESSES IN INDIAN JOURNALS}

Reporting on ethical processes in two Indian pediatric journals in this issue of Indian Journal of Medical Sciences, Bavdekar et al. ${ }^{[1]}$ have concluded that a significant proportion of articles published in these two journals have not provided information regarding obtaining of ethical approval, written informed consent, and assent; and imagine, I am asked to write a commentary on this article (or defend myself), being the editorin-chief of one of the journals in question!

Bavdekar et al..$^{[1]}$ have raised a valid issue, and their findings are in conformity with studies from the rest of the globe indicating that the problem is not limited to Indian journals alone. On a positive note, I am rather happy that ethical clearance is reported for more than one third of the prospective studies, despite the fact that the authors were not being guided by the journals on reporting of ethical processes. I am sure that once journals take up these issues and incorporate them in their instructions to authors, reviewers' pro forma sheet, and checklist for authors, matters will improve. But till then, who is to be blamed for the current dismal reporting of ethical processes: editors, who publish these studies without verifying whether ethical approval and written informed consent/assent was taken or not; reviewers, who do not give appropriate importance to this important information while sending their recommendations; or the authors, who fail to obtain/report the desired ethical permissions? Everyone will have one or the

I agree with the authors that the results other excuse [Figure 1]

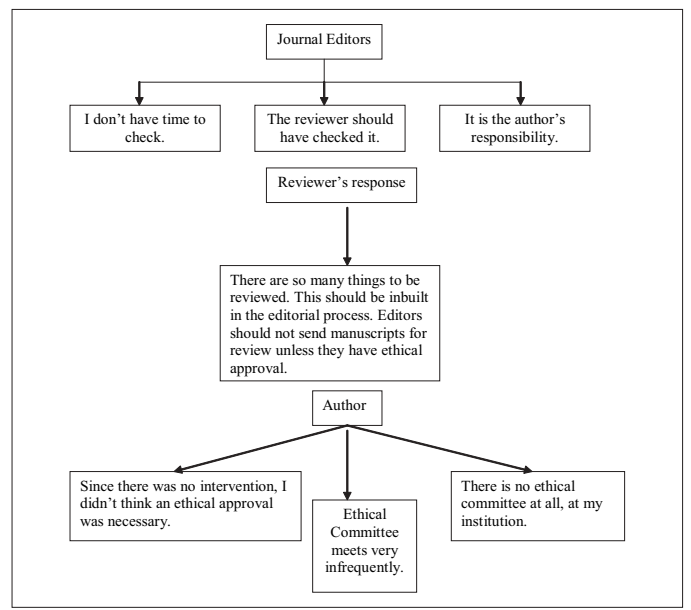

Figure 1: Excuses offered for lack of reporting of ethical research

can be extrapolated to other biomedical journals in India. However, one of the major limitations of the study was that the individual authors were not contacted to determine the reason(s) for nonreporting of ethical processes. This mattered a lot. It would have not only documented the discrepancy between what was done and what was reported but also the reasons thereof. Absence of a statement does not automatically imply that consent was not taken. Many a times due to shortage of space (for example, letter to editor) or ignorance of writing style, this may not be mentioned. On the other side (for manuscripts which reported adherence to ethical processes), it was also important to determine whether the process of such reporting in itself was ethical or not.

\section{MISSED OPPORTUNITIES}

There is a lack of exposure to culture of reporting medical research (ethics, in particular), starting right from the undergraduate days in the medical school. Even later, such opportunities are far and few in between. As a result, most of the researchers in India are ill informed about, and poorly equipped with, the process for obtaining ethical clearance, access to tools for taking consent, and requirements for assent, etc.

During undergraduate days, though a medical student learns about medical ethics, consent, and assent while studying forensic medicine, there is hardly any emphasis, during clinical training, on its application. Also, students are not supposed to do research. In fact, it is discouraged; an undergraduate medical student I closely know drew up a research project, only to be told by the concerned authorities, 'This is not your job, you only study, write the exam, get the degree, and then do whatever. No wonder, the flowers don't bloom. A laudable initiative is the Indian Council of Medical Research (ICMR) Student Fellowship that promotes research by medical students and also exposes them to the tools of research methodology, including ethical considerations. However, the proportion of students applying for and ultimately executing such research is very low, compared to the total number of medical undergraduate students in India.

Every postgraduate student is supposed to write a dissertation/thesis before the degree is awarded. Most of the time, the protocols are presented, discussed, and approved in departmental/institutional meeting and ethical clearance is presumed. There is no formal approval of the institutional review board (IRB), because the IRB either does not exist or remains nonfunctional. Of the more than 250 medical colleges in India, not more than 20 (a rough estimate) have a properly constituted institutional advisory board/ethical committee 
as per ICMR guidelines.

Postgraduate courses are now also conducted at hospitals/institutions other than those affiliated to medical colleges i.e., Diplomate of National Board, which often do not have a valid or set process for granting ethical clearance. Also, if a private practitioner contemplates research on an individual basis, what is the process or facility for obtaining ethical permissions?

\section{THE WAY FORWARD}

Editors of biomedical journals should ensure appropriate reporting of ethical processes prior to accepting articles for publishing. The policy of the journal on this aspect should be clearly spelt out and widely disseminated. Reviewer pro forma should have a specific column for commenting upon ethical processes; similarly, checklist for authors should be augmented by adding a checkpoint each, for reporting ethical clearance and informed consent/assent. Needless to say, Indian Pediatrics has already taken these precautions. It would be interesting to conduct this study again 2 years later and compare the results.

The long-term solution consists of exposure to ethic-based research culture right from day one in medical school. A commendable initiative of the Medical Council of India in improving medical education was to make it mandatory to establish medical education unit at every medical college in India. An apex body such as ICMR can promote research by medical students on similar lines. A medical research cell equipped with an institutional ethics committee (constituted as per ICMR guidelines) should be a must in every teaching institution accredited for conducting undergraduate or postgraduate medical courses in India. A medical student should be encouraged to complete at least one research project during the entire training; this should also be weighted for final evaluation/grading. Ethical clearance should be a prerequisite for all postgraduate dissertations/theses before the work is approved. Indian Medical Association is the largest body of physicians in India. Indian Medical Association in collaboration with professional organizations of other specialties may establish IRB in every city to cater to the needs of private practitioners who desire to conduct research but do not have access to the ethical committee of teaching institutions or are hesitant to approach them.

\section{REFERENCE}

1. Bavdekar SB, Gogtay NJ, Wagh S. Reporting ethical processes in two indian pediatric journals. Indian J Med Sci 2008;62:134-140.

PIYUSH GUPTA

Professor of Pediatrics, University College of Medical Sciences, Delhi; and Editor-in-chief, Indian Pediatrics, Block R-6-A, Dilshad Garden,

Delhi - 110 095, India

Correspondence: E-mail: drpiyush@sify.com 